\section{DGG-Arbeitsgruppe kritisiert Empfehlungen zur Zoster-Impfung}

\begin{abstract}
Die Arbeitsgruppe Impfen der Deutschen Gesellschaft für Geriatrie (DGG) sieht die aktuellen Empfehlungen der Ständigen Impfkommission (STIKO) zur Zoster-Impfung skeptisch.
\end{abstract}

Estongen wird keine allgemeine Anwendung - des Herpes-Zoster-Lebendimpfstoffs als Standardimpfung zur Verhütung von Herpes Zoster, seinen Komplikationen und Spätfolgen beim Erwachsenen empfohlen, berichtete Dr. Anja Kwetkat, Sprecherin der DGG-Arbeitsgruppe und Chefärztin der Klinik für Geriatrie am Universitätsklinikum Jena.

Wesentlicher Grund für die Nichtempfehlung der STIKO sei, dass die Wirksamkeit der Impfung mit zunehmendem Alter abnehme. Dabei haben aber gerade hochaltrige Patienten ein deutlich erhöhtes Risiko, an Herpes Zoster zu erkranken. Auch die Schwere der Erkrankung nimmt mit dem Alter deutlich zu. „Wir halten eine Impfung mit dem attenuier- ten Lebendimpfstoff entsprechend der Zulassung auch für alte Menschen für sinnvoll“", sagte Kwetkat.

Post-Zoster-Neuralgie (PZN) tritt bei Älteren besonders häufig auf Die PZN tritt besonders häufig bei Älteren auf, heißt es in der Stellungnahme der DGG. Die Zoster-Schmerzen können trotz adäquater Therapie über Monate, teilweise über Jahre persistieren und die Lebensqulität stark vermindern. Die Schmerzen sprechen häufig auch auf eine Opiattherapie nicht ausreichend an. Laut der Stellungnahme wurde die durch die Impfung verbesserte Lebensqualität durch Verhinderung der Erkrankung in der gesundheitsökonomischen Analyse der STIKO nicht berücksichtigt.Aus Sicht der DGG ist eine Wirksamkeit der Impfung von $41 \%$ bei den 70 - bis 79 -Jährigen zwar nicht optimal. Berücksichtigt man die hohe Inzidenz der Erkrankung in dieser Altersgruppe, können durch die Impfung trotzdem viele Fälle von Herpes Zoster und damit auch viele Neuralgien verhindert werden.

Der Impfschutz hält bis zu sieben Jahre an, was aus Sicht der AG Impfen der DGG im Vergleich zu vielen anderen Impfungen bei alten Menschen eine ausreichende Schutzdauer ist. Eine BoosterImpfung nach zehn Jahren führt zu einer ähnlich starken Antikörperbildung und einer höheren Zahl an VarizellaZoster-spezifischen Memory-Zellen als bei Menschen, die das erste Mal gegen Herpes Zoster geimpft wurden. Die AG Impfen der DGG hält eine Impfung mit dem attenuierten Lebendimpfstoff entsprechend der Zulassung auch für alte Menschen für sinnvoll.

Pressemeldung der Deutschen Gesellschaft für Geriatrie vom 27.09.2017

\section{Fahrtauglichkeit: Individuelle Gesundheitschecks für Senioren}

Forderungen, wonach das Autofahren ab einem bestimmten Alter nur noch mit einem Leistungszertifikat oder verpflichtenden Gesundheitschecks erlaubt sein soll, erteilt die Deutsche Gesellschaft für Geriatrie (DGG) eine Absage. „Dieses pauschale Vorgehen ist aus medizinischer Sicht grundsätzlich abzulehnen", sagt der DGG-Präsident Prof. Jürgen M. Bauer.

Z ahlen des ADAC belegen: Lediglich $15 \%$ der Pkw-Fahrer ab 65 Jahre sind Hauptverursacher eines Unfalls mit Personenschaden. „Um wirklich einen Beitrag zur Sicherheit im Straßenverkehr zu leisten, sind gerade im Alter regelmäßige und vor allem individuelle Gesundheitschecks unumgänglich", sagt der DGG-Fahrtauglichkeitsexperte Dr. Dirk Wolter, Chefarzt der Abteilung Gerontopsychiatrie an der LVR-Klinik Bonn.

Eine verpflichtende Einführung von Gesundheitschecks unterstützt Wolter nicht: „Das muss von Fall zu Fall individuell betrachtet werden." Zudem müsse

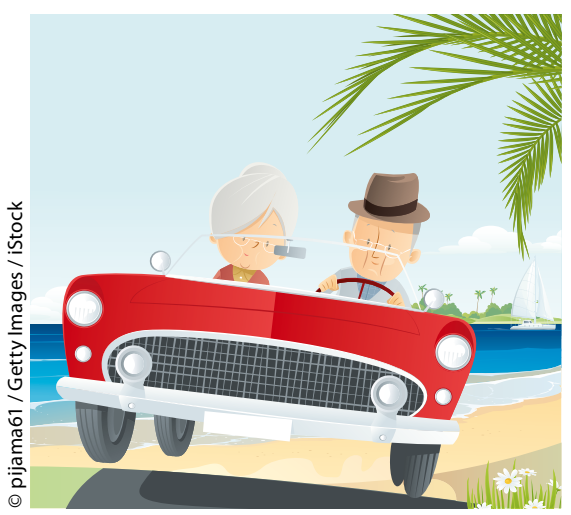

Mit einem freiwilligen Gesundheitscheck auch im Alter sicher Autofahren. genau geklärt werden, welche Untersuchungen ein solcher Test im Einzelfall abdecken soll. Schon ein Sehtest für ältere Kraftfahrer müsse ganz andere Bedingungen erfüllen als für junge Fahrer. Wolter weist auf eine Reihe von Arbeitsmaterialien hin, die Medizinern beim Abklären der Fahreignung im höheren Lebensalter helfen sollen. Diese stehen zum kostenlosen Download auf der Homepage der Deutschen Gesellschaft für Gerontopsychiatrie und -psychotherapie zur Verfügung.

\section{Verlust von Autonomie und Selbstbewusstsein verhindern} Das Ziel aller beteiligen Mediziner müsse es sein, die Mobilität der Älteren so lange wie möglich zu erhalten. „Andernfalls verlieren viele Menschen zu früh an Autonomie und Selbstbewusstsein, was im Alter schwerwiegende Gesundheitsfolgen haben kann", sagt der Experte.

Pressemeldung der Deutschen Gesellschaft für Geriatrie vom 29.11.2017 